\title{
Analysis of the Ancient Papermaking Technology from Qiliang Village, Shaanxi Province
}

\author{
Qi Gao ${ }^{1, *}$, Dayong Jiang ${ }^{2}$, Xiangwu Meng ${ }^{3}$ \\ ${ }^{1}$ Weifang University, Weifang of Shandong Province, China, 261000 \\ ${ }^{2}$ Engineering University of Pap, Xi'an of Shaanxi Province, China, 710000 \\ ${ }^{3}$ Lanzhou University of Technology, Lanzhou of Gansu Province, China, 730000
}

Keywords: papermaking culture; Qiliang ancient papermaking; Cailun-paper; inheritance and innovation

\begin{abstract}
Papermaking is one of the four great inventions in China. The ancient papermaking technology from Qiliang Village (also named as "Cailun-Paper") has over a thousand-year history and its own characteristics. In 2011, Qiliang Ancient Papermaking Technology was enlisted in the National Intangible Cultural Heritage Catalogue. This study systematically summarizes the origin, development and current status of Qiliang Ancient Papermaking Technology in chronicle order; In addition, it records and analyses the raw materials, tools and technological process of Qiliang Ancient Papermaking Technology; In the end, this study tries to evaluate the future value and explore the modern inheritance and innovation utilization of Qiliang Ancient Papermaking Technology
\end{abstract}

\section{Introduction}

Located in the northeastern region of Zhouzhi County, Shaanxi Province and to the south of Qinling Mountains, Qiliang Village has advantages in its superior geographical location [1]. Since the Han Dynasty, Qiliang Village has specialized in the production of handmade paper. Until now, Qiliang Village Ancient Papermaking Technology has a history of around 2000 years. The handmade paper of Qiliang Village is also named as Cailun Paper (or Hemp Paper) because its papermaking technology is originated from Cailun Papermaking Technology. Qiliang handmade paper has excellent quality. It has natural raw edge. Besides the variety of appearance color, the paper thickness also varies. Since Qiliang paper is handmade without any chemicals added in the production process, it poses no harm and pollution to the ecological environment. Since the 1980s, Qiliang Ancient Papermaking Technology has been negatively impacted by the mass development of industrial papermaking technology. It almost faced the danger of decline. Fortunately, in 2011, Qiliang Ancient Papermaking Technology was included in the National Intangible Cultural Heritage Catalogue. Its cultural value and historical significance attracted widespread attention of the society since it received the support from government to recover the development of the technology [2].

\section{The Origin of Qiliang Ancient Papermaking Technology}

Before paper was invented, the ancients first recorded things by knotting ropes or stacking stones. Later, symbols and characters were depicted on trees or pottery, or on shells, keels, tortoise backs and stones [3-5]. With the development of society, bamboo slips, parchment books and silk books appeared, which help to record the culture and history in a more advanced way. However, bamboo slips and parchment books also have the drawbacks as they are not easy to carry and preserve. Only until paper was invented, a new way of life and production appeared.

\subsection{The Origin of Ancient Papermaking Technology}

The dispute on the emergence, invention and representative of papermaking technology has not 
been resolved by the papermaking industry and academia. According to Cai Lun's Biography in the Later Han Dynasty Historical Records, most of the paper before Cailun was silk. Paper was invented by Cailun when the Emperor He of Han was in power. Cailun had the idea to look for better writing material when he saw the ministers were inconvenient in using bamboo slips. He summarized the silk and leather paper making process and attempted to utilize bark, hemp, rag head and other raw materials to make paper. He tried to soak, cook, mash and many other steps to process the above-mentioned raw materials into serous fluid. Afterwards, he spread the serous fluid on the flat mat. A thin layer of paper formed when the serous fluid was dried. However, there are some other historical records saying paper was in existence 30 years before Cailun invented his papermaking technology [6]. Whether Cailun was the inventor of paper or not, his contribution to papermaking can not be ignored. He improved papermaking technology and promoted the usage widely. The papermaking technology in Qiliang Village mainly follows Cailun Papermaking Technology. Therefore, Qiliang ancient handmade paper is often called Cailun paper. (Figure 1)

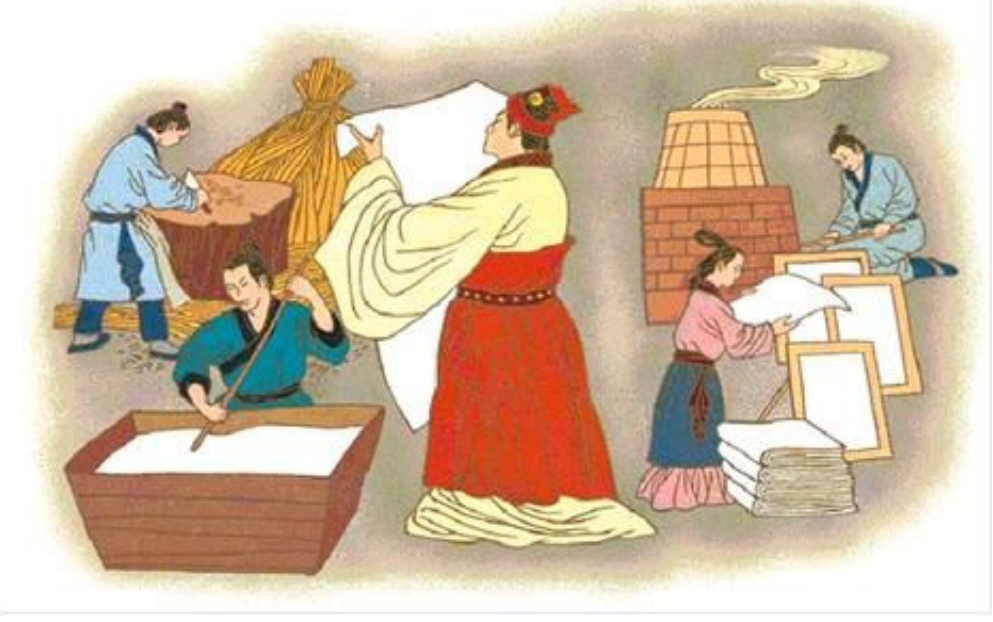

Figure 1 CaiLun Paper Making Scene in the Eastern Han Dynasty (Picture from the Internet)

\subsection{The Development History of Qiliang Ancient Papermaking Technology}

Qiliang Village is located to the north of Qinling Mountains andthe Baima River crosses the village. At the earliest time, there were only three families in the village, surnamed Liu, Zhang and Zhou, all of whom were engaged in papermaking business. Cai Lun Papermaking Technology was complex and required much manpower. It wasnot easy for a family to complete the whole papermaking process alone. Therefore, thethreefamilies united and helped each other to produce paper together. Eventually, theydevelopedQiliang Village into a papermaking centric village. Qiliang Papermaking Technology achieved the height of its fame in Han Dynasty [7]. People from Qiliang name the mills that produce paper as Paper Han Mill, the paper-fishing pond as Paper Han Pond and the stone board on the paper fishing pond as Paper Han Stone. According to historical records, most of the paper used in the Imperial Palace in the Han Dynasty was produced and supplied by Qiliang Village. The quality of the Cailun Paper from Qiliang was superior and did not deteriorate after thousands of years. Therefore, Qiliang paper was always in short of supply on the market (Figure 2). However, with the change of writing tools and the emergence of industrial papermaking, the ancient method of papermaking declined gradually. Especially in the 1960s and 1970s, the Qinling Mountains were closed for war, resulting in insufficient supply of papermaking raw materials, such as bark. Coincided with drought and water shortage at the same time, Qiliang Village was once not able to produce any paper. Since the beginning of the 21st century, the Chinese government attaches great importance to the cultural heritage protection. Qiliang Papermaking Technology attracted wide attention from public and regained its vitality after it was listed as the National Intangible Cultural Heritage. 


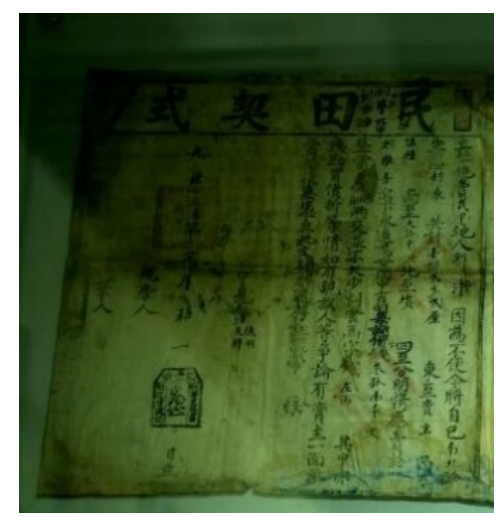

Figure 2 The Qing Dynasty Land Deed thatUsed CailunPaper (Source: author's photography)

\subsection{Current Situation of Qiliang Village Papermaking Technology}

Currently, there are 12 old craftsmen from Qiliang Village who have mastered the ancient papermaking technology (Figure 3). In order to protect and carry forward the traditional handicraft papermaking technology, Liu Xiaodong, a retired teacher from Qiliang Village, convened all of the old craftsmen to reestablish a Cailun Paper Workshop. Currently, the Cailun Paper Workshop has been transformed into Cailun Paper Culture Centre [8].

Liu Xiaodong and others have not only respected and inherited the revival of ancient papermaking. They have created precious Panda Paper based on the feces of giant pandas from Qinling Mountains, the bark of tussah trees and kiwifruit vines. Panda Paper is a high-quality painting and calligraphy material with the advantages of bamboo paper and bark paper. Panda Paper is thick, anticorrosive and has natural bamboo fragrance. The bold innovation of Qiliang Village to turn waste into treasure has fully reflected the excellence of Chinese traditional culture. It cannot be neglected that the traditional papermaking still faces difficulties in development. The raw material and labor costs are quite high. Thus, even though the price of the paper is $40 \mathrm{RMB}$ each, the profit is hard to cover all the costs. Few and few young generations are engaged in ancient papermaking, leading to the concern of inheritance of ancient papermaking in modern society.

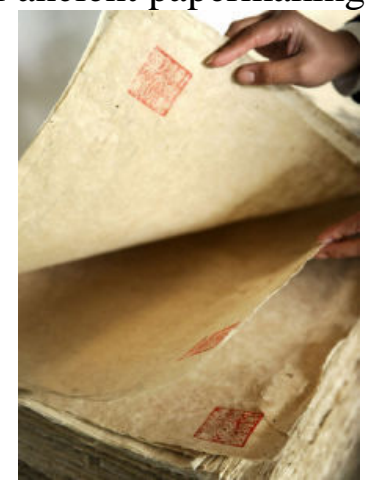

Figure 3 Qiliang CailunPaper for Sale (Source: Pictures from the Internet)

\section{Qiliang Ancient Papermaking Technology}

The difference between the traditional CailunPaper and the innovative PandaPaper from Qiliang Village is mainly reflected in the raw materials and the composition ratio of raw materials. Both categories of paper have same papermaking process.Below paragraphs will take the traditional Cailun Paper as the research object and analyze the ancient papermaking technology from raw material, tools and processes perspectives [9].

\subsection{Raw Material of CailunPaper}

As can be confirmed from various historical records, cultural relics analysis and the description by the existing craftsmen, the main raw material of Qiliang Ancient Papermaking Technology is the bark of paper mulberry from Qinling mountain [10]. There are a lot of lignin, pectin and fibers in 
the bark of paper mulberry, which just fits the demand of papermaking raw material. (Figure 4)

The bark can be subdivided into bud bark and winterbark.Bud bark is not often used. Itiscollected in spring when the paper mulberry tree germinates. This kind of bark has less fibre, low paper conversion rate and poor quality. Winter bark refers to the autumn and winter bark, which has more fibers, high paper conversion rate and light herbal aroma. Therefore, winter bark is the main raw material for making Qiliang Cailun Paper. Besides, due to the limited source of the bark and the complexmaking requirements, the output of Cailunpaper is not very high. However, the produced paper quality is very high and is suitable for painting and calligraphy.

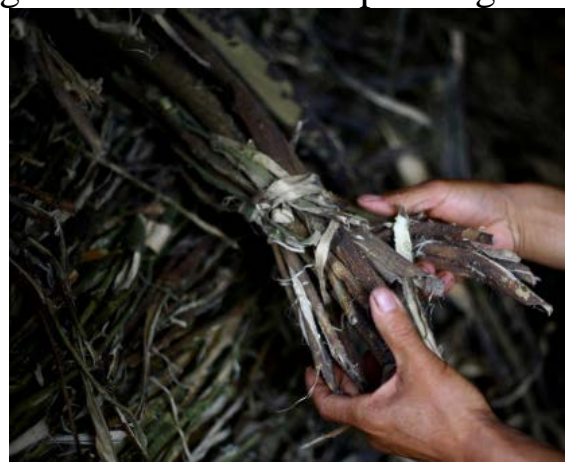

Figure 4 A Bundle of Bark to be used (Source: Picture from the Internet)

\subsection{Tools to Produce Cailun Paper}

The tools involved in papermaking are relatively simple which include washing pool, big steamer, cutter, hammer, pulp pool and papermakingmould,etc.(Figure 5) The key step of ancient papermaking is making pulp and paper molding. After the bark is boiled in a big steamer, it will be cut into uniform pieces and then pour into the pool to stir thoroughly. The excess moisture will be filtered out by a papermaking mould, leaving only a thin layer of pulp slices on the mould. The pulp slices will become paper after being dried. In addition to the pulp that influences the quality of paper, paper molding will also play a significant role in the final quality of paper. Paper molding is a kind of mold. [12] When the pulp is stirred thoroughly in the water, a paper molde will be placed in a diagonal arc into water to make the pulp stick to the slice evenly. This method is called paper molding. The major tools utilized in the molding process include frame and papermaking mat.

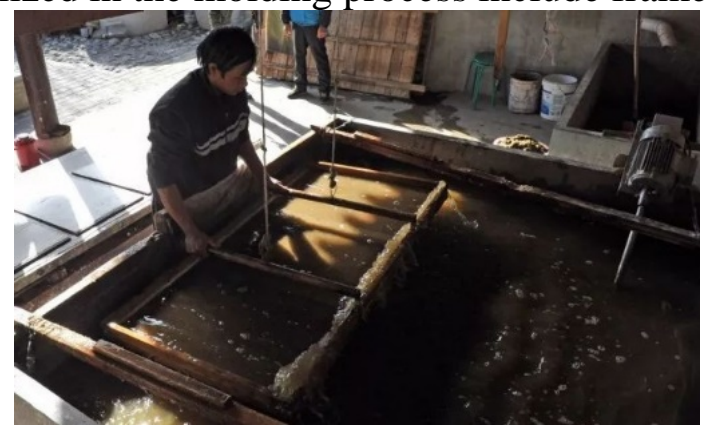

Figure 5 Pulp Pool and Papermaking Mould

In order to improve the drainage performance of papermaking moulds, the wise Qiliang people, after a long period of practice, used fine silk materials such as horsetail. They wove the material into crisscross mesh screens with small holes and fixed the mesh screens on the edge of the mould frame. The paper made from this process will have warp and weft patterns. The unearthed paper cultural relics indicated that the paper made from Han Dynasty to Jin Dynasty are all wove paper. There are also differences in the size of paper in different historical time. In addition, Qiliang people also came up with the idea of making paper mats with slim bamboo splints, and the paper mat can be flexibly disassembled. This development not only solves the dilemma that a set of mould can only make one piece of paper, but also solves the problem that wet paper isdifficult to form into shapes. Bamboo paper mat reduces manpower, time to produce paper and significantly improves the production efficiency. The use of bamboo splints mat is an important breakthrough in 
the history of papermaking tools.

\subsection{Technological Process of Cailun Paper}

The production process of Qiliang Cailun Paper is relatively complex, which includes36 major processes and 72 minor processes. It has been known as 108 processes to make a CailunPapersince ancient times. From the perspective of technological process, the formation of a piece of paper requires three steps: paperpulp, pulp sheet and paper drying. The whole process can be divided into nine major processes: cutting, washing, soaking, boiling, pounding, making pulp, molding, sun-drying and paper uncovering.In addition to the nine major processes, dozens of small processes are needed to complete the production of a piece of paper. The entire process will take about a month.

Each process has strict operating rules, and slight errors may cause the paper to fail its production.

After obtaining the raw material of the bark of the tussah tree, the first step is to immerse the bark in the pond. The temperature of the water increases when the sun shines, leading to the microorganisms reproduce rapidly[13]. These microorganisms feed on pectin in the bark. Therefore, the pectin in the bark of the tussah tree can be removed with the increase of microorganisms. However, it is gradually found that the extraction method is too time-consuming. Thus, edible alkali is added to the pond to accelerate the degumming of the bark. At the same time, edible alkali can also decompose lignin and form the fibers that are necessary in the papermaking production process. Next, the selected hundreds of kilograms of the bark will be steamed twice before and after being put into a larger pot. It will be first steamed for 7 hours, and then dried. Afterwards, it will be soaked in the pond. Several days later, it will be steamed and soaked again. As a result, the plant fibers in the bark of the tree are fully softened, and some impurities in the bark can be filtered out. Cailun Paper requires a high steam technology, and the price of steamed bark is relatively expensive. During the Southern and Northern Dynasty, the price of one mu of the bark of the tree is close to the priceof ten pieces of silk. The quality of the paper is good and the paper is durable. Therefore, the Qiliang people insisted on making the paper. After steaming and softening, the materials will be stepped on by the craftsmen. The black spots and impurities on the bark surface will be removed by the repeated stampede. After steaming, the bark fibers can not be directly pulped. Because these fibers also have a shell which will cause the fibers to not coagulate together. The bark must first be crushed into a cake shape (Figure 6), and then cut the bark (Figure 7). Afterwards, the multi-layer bark stacked is evenly cut with a big knife. After thoroughly pounding and making the pulp in the paper-making pool, the pulp water is formed. After subsequent papermaking, paperpressing and sun-drying (Figure 8), Cailun Paper is eventually formed. (Table 1)

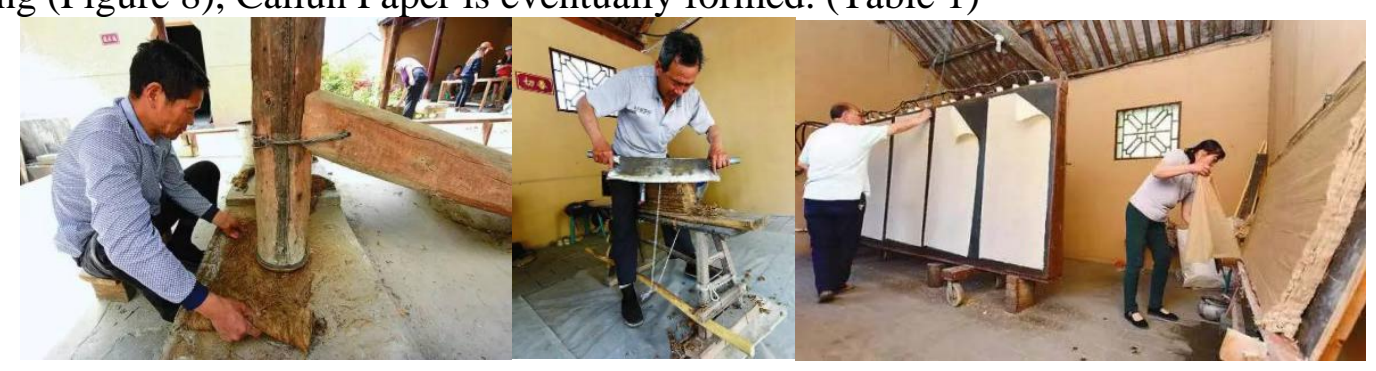

Figure 6 Bark Pounding

Figure 7 Bark Cutting

Figure 8 Paper Drying

It is difficult to make large paper and thin paper based on Qiliang Village Papermaking Technology. Thin paper requires high quality pulp and excellent papermaking skills of craftsmen. The key to the uniformity of paper thickness lies in the process of paper fishing. The craftsmen hold bamboo curtain in water and judge the angle of entry and the speed of picking up according to the water flow. The production of large paper requires many craftsmen, large enough papermaking ponds and papermaking molds. At present, the maximum size of the paper Qiliang Village can produce is six feet. If larger size is needed, paper stiching is neede 
Table 1 Table of Ancient Paper Making Process

\begin{tabular}{|l|l|l|}
\hline process & \multicolumn{1}{|c|}{ mode of operation } \\
\hline washing & $\begin{array}{l}\text { Wash and soak the bark in } \\
\text { water to soften it }\end{array}$ \\
\hline soaking & the bark more softer. \\
\hline boiling & $\begin{array}{l}\text { boil in a steamer to soften } \\
\text { bark and decompose }\end{array}$ \\
\hline pounding & & $\begin{array}{l}\text { Remove residual impurities } \\
\text { from bark }\end{array}$ \\
\hline paper & $\begin{array}{l}\text { Put the cutted bark into the } \\
\text { mortar and pound them } \\
\text { repeatedly. }\end{array}$ \\
\hline making pulp & & $\begin{array}{l}\text { Soak and soften the mashed } \\
\text { bark and pulp it. }\end{array}$ \\
\hline
\end{tabular}

Source: Renovation of Qiliang Village's Ancient Paper-making Technology and Research on the Excavation and Protection of Residential Environment

\section{Conclusion}

Paper is one of the four great inventions in China. Its traditional cultural value and historical importance cannot be ignored. After the restoration of Qiliang Village Ancient Papermaking Technology, it attracted widespread attention and recognition from public. Because of its excellent paper quality, it was especially purchased by the calligraphers and painters. Additionally, Qiliang Village has been awarded the first batch of excellent social practice bases for traditional culture education in primary and secondary schools in Shaanxi Province. In recent years, more than 20,000 students from all over the country have visited Qiliang. By getting to know the history and connecting to the current papermaking, students have approached the traditional literature with its modern values. More importantly, the production technology of Cailun Paper has been filmed in documentaries by CCTV and many other media. Qiliang Ancient Papermakingis a low-carbon and environmental-friendly production process, which has attracted the study interests of researchers from home and abroad. In terms of the technology innovation, Qiliang Village has developed a high-end product of Panda Paper.The feces of giant pandas consisted of 70\% bamboo fiber. Qiliang Village has successfully utilized the traditional techniques to create a premium paper based on the waste from the national treasure panda. This study systematically summarizes the Qiliang 
Papermaking Technology based on a deep dive of ancient papermaking techniques. However, the traditional process of ancient paper-making still faces many difficulties in inheritance and protection. Such problems as limited target customers, no successors to takeover the production techniques, the time-consuming process and limited economic benefits still concern the future development of Qiliang Papermaking Technology. In the future, studies will be carried forth to understand how to further inherit the ancient papermaking technology, develop the craftsmen to takeover the precious technology and how to effectively promote and innovate such traditional products and balance the challenges from mass industrial papermaking. All these questions are key to inherit and protect the Qiliang Ancient Papermaking Technology.

\section{Acknowledgements}

This paper is supported by the National Natural Science Foundation of China (NO.51568038) and Science and Technology Development Plan of Weifang (2018GX005).

\section{References}

[1] Wang Jingyi. Study on the excavation and protection of ancient paper-making and residential environment in Qiliang Village, Xi'an: Xi'an University of Architectural Science and Technology, 2017.

[2] Shi Yujie. Research on the Protection and Development of Ancient Paper Making by Zhou Zhiliang, 2017 (14): 7-9.

[3] Fan Shengjiao. Path Study on the Transition from Non-material Cultural Heritage to Non-material Economic Industry --- Taking Shiqiao Ancient Paper Making in Guizhou as an Example. Journal of Kaili College, 2013 (01): 50-53.

[4] Cai Fengming. Cultural Characteristics and Contemporary Value of China's Intangible Cultural Heritage, Journal of Shanghai Jiaotong University (Philosophy and Social Sciences Edition), 2006 (04): 64-69.

[5] Yang Haozhong and Zhang Weiping. Integral protection of intangible and material cultural heritage - Fengxiang clay sculpture and its architectural environmental protection in Shaanxi. Architecture and Culture. 2006 (07): 13-16.

[6] Liu Yi, Sunshine. Ancient Paper Making: Bright Business Card of Cultural Confidence --Exploration of Caihou Paper Cultural Park in Qiliang, Xi'an. Shaanxi Daily, 2017.05.19 (13): 01-05.

[7] Zhou Wenjuan. The Role of New Media in the Development of Traditional Handicraft Industry --- Take the Paper Industry of Ancient France as an Example. Young Journalist, 2017 (03): 93-94.

[8] Hu Xiaozhong. The birth of paper promotes the development of world civilization. Cai Lun's contribution to human beings by inventing papermaking. Vocational education, 2017 (09): 24-25.

[9] Zhang Limin, Self-consistent Evolution: An Analysis of the Necessity of Rational Commercialization of Non-legacy: Taking Traditional Handicraft as an Example, Folklore Research, 2014 (02): 11-12.

[10] Department of Non-material Cultural Heritage, Ministry of Culture, Compilation of Laws and Regulations on the Protection of Non-material Cultural Heritage, Beijing: Culture and Art Publishing House, 2013.

[11] Luo wenbo, Research on the Modernization Construction of Chinese Handmade Paper-Based on the Cultural Thinking of Typical Handmade Paper Field Survey. Hefei: China University of Science and Technology, 2017.

[12] Yang Juzhong. The Origin of Ancient Chinese Paper-making Law and Cai Lun's Position in 
Paper-making History. Journal of Shaanxi Normal University (Philosophy and Social Sciences Edition), 2001, (03): 153-156. $\square$

[13] Franklin.Description of the process to be observed in making large sheets of paper in the Chinese manner, with one smooth surface. Transactions of the American philosophical Society (Philadelphia), 1793:8-10. 